\title{
Low-Complexity MMSE Channel Estimator for MB-OFDM UWB Systems
}

\author{
Moufida Hajjaj, Fadoua Mhiri, and Ridha Bouallegue
}

\begin{abstract}
Channel estimation is one of the key technologies in multi-band (MB) Orthogonal Frequency Division Multiplexing (OFDM) Ultra Wide Band (UWB) systems, which has received more and more consideration. MMSE channel estimation has been known as superior performance channel estimation. However, this algorithm has high computational complexity. In this paper, a modified MMSE channel estimation for MB-OFDM UWB is proposed. We evaluate the performance of the modified channel estimator in terms of the bit error rate (BER) by computer simulations. It is shown that the proposed channel estimator gives the best tradeoff between performance and complexity.
\end{abstract}

Index Terms-MB-OFDM, UWB, OLR-MMSE channel estimator.

\section{INTRODUCTION}

During the past few years, Ultra-wideband (UWB) has promised as an efficient technology for future wireless short-range high data rate communication. Federal communications commission (FCC) has already allocated the spectrum from $3.1 \mathrm{GHz}$ to $10.6 \mathrm{GHz}$ for UWB applications. Due to its large bandwidth UWB has the promise of high data rates [1].

In 2008 the WiMedia Alliance [2] working with ECMA (the European Computer Manufacturers Association) introduced the WiMedia MB-OFDM (Multiband Orthogonal Frequency Division Multiplexing) UWB radio platform as their global UWB standard. According to it, the available spectrum $(3.1 \mathrm{GHz}-10.6 \mathrm{GHz})$ is divided into 14 sub-bands. Each sub-band of $528 \mathrm{MHz}$ offers $480 \mathrm{Mbit} / \mathrm{sec}$. In order to introduce multiple accessing capabilities and to exploit the inherent frequency diversity, each OFDM symbol is transmitted on a different sub-band as dictated by a time-frequency code (TFC) that leads to band hopping [3]. Thus MB-OFDM is considered to be the pioneer implementation approach for UWB technology [4].

Further, an important block in the transmission chain is the channel estimator which is very important issue for coherent MB-OFDM UWB systems. Different type of pilot arrangement as comb-type and block-type are presented in the literature [5].

According to The first kind of pilot arrangement, the pilot signals are uniformly distributed within each OFDM block. Since only some sub-carriers contain the pilot signal, the channel response of non-pilot subcarriers will be estimated by

Manuscript received October 2, 2013; revised December 3, 2013.

The authors are with the Innov'COM Laboratory research in telecommunication at Higher School of Communications of Tunis, Tunisia (e-mails: moufida.hajjaj@gmail.com, mhiri_fadoua@yahoo.fr, ridha.bouallegue@gmail.com). interpolating neighboring pilot sub-channels. Thus, the comb-type pilot arrangement is sensitive to frequency selectivity when comparing to the block-type pilot arrangement system. Where, the pilot signal is assigned to a particular OFDM block, which is sent periodically in time domain. This type of pilot arrangement is especially suitable for slow-fading radio channels [6]. Therefore, this study investigates the block-type pilot channel estimation for multi-band orthogonal frequency division multiplexing (MB-OFDM) systems.

The channel estimator response is usually obtained by either LS or MMSE estimates of training pilots. The MMSE estimator produces much better performance than LS estimators. A major drawback of the MMSE estimator is its high computational complexity, especially in matrix inversions. To overcome this drawback, the optimal Low-rank MMSE channel estimator for MB-OFDM UWB system is proposed to reduce complexity.

The rest of the article is arranged as follows: Section II gives base band implementation of MB-OFDM UWB system and the channel model. The implementation of OLR-MMSE and other algorithms (like LS and MMSE) is discussed in Section III. Section IV describes the performance analysis of different algorithms in term of complexity and BER, and finally Section V draws up the conclusions.

\section{SYSTEM MODEL}

Fig. 1 illustrates the transmitter and the receiver of the MB-OFDM UWB system. They consist of two parts: baseband and radio frequency (RF). The baseband of the transmitter consists of a scrambler, a convolutional encoder, a puncturer, a bit-interleaver, a constellation mapper, and a block of an OFDM modulation and add Cyclic Prefix (CP). After baseband processing, signal is transmitted through UWB channel. The baseband of the receiver, in general, consists of similar blocks of the baseband in the transmitter but in the reverse order [7].

\section{A. Transmitter Structure}

The baseband of the transmitter as shown in Fig. 1 (a) consists of a data scrambler, a convolutional encoder and puncturer, a bit-interleaver, a constellation mapper, and OFDM modulation.

1) The scrambler is used to convert data bit input sequence into a pseudo random sequence. The polynomial generator of the pseudo random binary sequence (PRBS) is: output=input $[n] \oplus$ input $[n-14] \oplus$ input $[n-15]$.

2) The convolutional encoder is based on the generator polynomials $g 0=\left[133_{8}\right], g 1=\left[165_{8}\right]$, and $g 2=\left[171_{8}\right]$. 


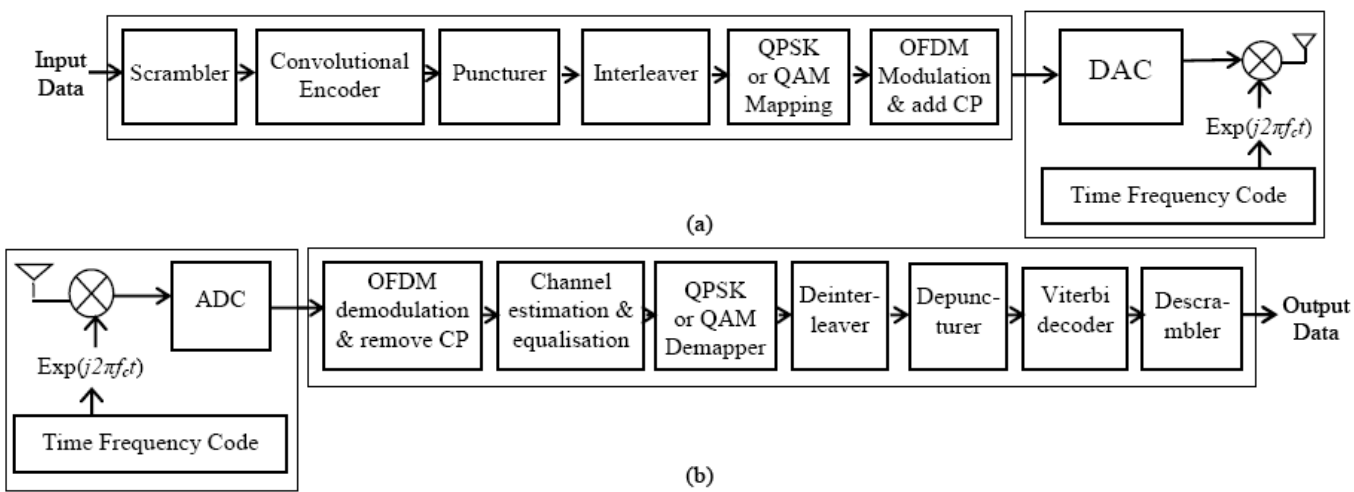

Fig. 1. The structure of a multiband OFDM UWB (a) transmitter and (b) receiver.

3) The convolutional encoder is based on the generator polynomials $g 0=\left[133_{8}\right], g 1=\left[165_{8}\right]$, and $g 2=\left[171_{8}\right]$.

4) Constellation Mapper is the fourth block in the baseband of the UWB transmitter. In this block, OFDM subcarriers are modulated using QPSK modulation or DCM modulation.

a) QPSK (quadrature phase shift keying) is used for data rates between $80 \mathrm{Mb} / \mathrm{s}$ to $200 \mathrm{Mb} / \mathrm{s}$.

b) DCM exploits channel diversity, for robustness against multi-path and interference, by adding an alternative form of redundancy for the higher data rates (from 320 to $480 \mathrm{Mb} / \mathrm{s}$ ).

5) MB-OFDM: the obtained symbols after OFDM modulation are then mixed up to the right sub-band, according to the time-frequency code (TFC). In this way, information is coded across all bands in order to exploit frequency diversity, and to provide robustness against multipath and interference.

The OFDM symbols transmitted in radio frequency (RF) signal utilizes a TFC (Time Frequency Code). There are two types of TFCs: Time-Frequency Interleaving (TFI), where the coded information is interleaved over three bands; and Fixed-Frequency Interleaving (FFI), where the coded information is transmitted on a single band [8].

Fig. 2 presents an example of a transmission in band group 1 with a TFC code [9]. the first symbol is transmitted on a center frequency of $3432 \mathrm{MHz}$, the second symbol is transmitted on a center frequency of $3960 \mathrm{MHz}$, the third symbol is transmitted on a center frequency of $4488 \mathrm{MHz}$, the forth symbol is transmitted on a center frequency of 3432 $\mathrm{MHz}$, and so on. Band group 1 (BG1) is a mandatory mode targeted for the first generation UWB devices.

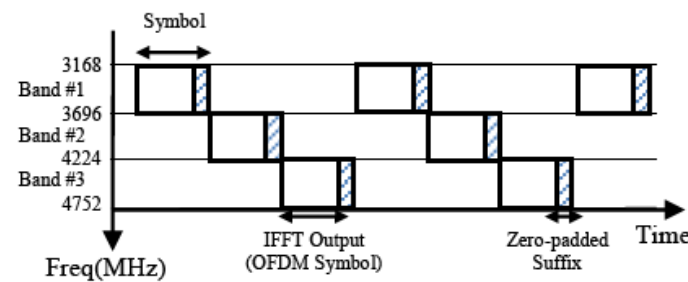

Fig. 2. OFDM symbols transmitted in RF signal utilizing a TFC within BG1.

\section{B. Channel Models}

The channel models chosen in simulation of MB-OFDM UWB communication system are standard IEEE 802.15.3a UWB channels considering realistic multipath resolution and operating frequencies. The models are derived from the Saleh-Valenzuela (SV) model with some minor modifications due to clustering phenomenon. There are four different UWB standard channel models, de noted as CM1, CM2, CM3, and CM4, which are based on the line-of-sight (LOS) multipath channel condition and the distance between the transmitter and the receiver [10].

1) CM1 corresponds to the LOS condition with distance of 0 to 4 meters

2) $\mathrm{CM} 2, \mathrm{CM} 3$, and CM4 are based on the non-LOS (NLOS) condition. The transmission distances in $\mathrm{CM} 2$ and $\mathrm{CM} 3$ are from 0 to 4 meters and from 4 to 10 meters, respectively. The transmission distance in CM4 is also from 4 to 10 meters but with an extreme NLOS condition.

\section{Channel Estimation TEChNiQues}

In block-type pilot based channel estimation, each subcarrier in an OFDM symbol is used in such a way that all sub-carriers are used as pilots. In this paper, the estimation of the channel can be performed either by the traditional estimators; Least Square (LS) and the Minimum Mean-Square Error (MMSE) or our optimal proposed Low-rank Minimum Mean-Square Error (OLR-MMSE) estimator.

\section{A. LS Channel Estimation}

In conventional comb-type pilot based channel estimation methods, the estimation of pilot signals, is based on the LS method is given by

$\hat{H}_{L S}(l)=X^{-1}(l) Y(l)=\left[\frac{Y(l, 0)}{X(l, 0)}, \frac{Y(l, 1)}{X(l, 1)}, \ldots, \frac{Y(l, N-1)}{X(l, N-1)}\right]^{T}(1)$

where:

The received signal $\boldsymbol{Y}(l)$ and the transmitted signal $\boldsymbol{X}(l)$ over UWB channel can be modeled by the following expression:

$$
Y(l)=X(l) H(l)+W(l),
$$

where $X(l)=\operatorname{diag}\{X(l, 0), X(l, 1), \ldots, X(l, N-1)\}$ in stands for the transmitted data symbol, $Y(l)=[Y(l, 0), Y(l, 1), \ldots, Y(l$, $N-1)]^{T}$ represents the received data symbol, $H(l)$ as in 
indicates the channel frequency response, and $W(l)=[W(l, 0)$, $W(l, 1), \ldots, W(l, N-1)]^{T}$ denotes the additive noise component, of the $l_{\text {th }}$ OFDM block. denotes the transposition operation.

Therefore, the LS estimator is susceptible to noise and Inter-Carrier Interference (ICI).Thus, the MMSE estimator has been shown to be better than the LS channel estimate in OFDM systems.

\section{B. MMSE Channel Estimation}

The MMSE channel estimate attenuations $H$, in (1), from the received data $Y$ and the transmitted symbols $X$ is

$$
\widehat{H}_{M M S E}(l)=R_{H(l) H(l)}\left(R_{H(l) H(l)}+\frac{\beta}{S N R} I\right)^{-1} \hat{H}_{L S}(l)
$$

where $\beta=E\left\{|X(l, k)|^{2}\right\} E\left\{|1 / X(l, k)|^{2}\right\}$ is a constant dependingon the training signal's constellation, $S N R=E\left\{|X(l, k)|^{2}\right\} / \sigma_{w}^{2}$ is the signal-to-noise ratio, $\sigma_{w}^{2}$ is the variance of AWGN and $R_{H(l) H(l)}$ denotes the auto-covariance matrix of the channel.

The MMSE estimator has better performance than LS estimation since it exploits the prior information on channel statistics. However, both estimators suffer from different drawbacks. The MMSE usually suffers from a high complexity level, where the mean-square error returned by LS estimator is high.

To reduce the complexity level and to improve the performance, in this paper, we propose an optimal low-rank approximation method that will be presented in the next section.

\section{Modified MMSE Estimator}

Modified MMSE estimators are studied widely to reduce complexity. Among them, an optimal low-rank MMSE (OLR-MMSE) estimator is proposed in this paper, which combines two simplification techniques.

First, a simplified MMSE estimation is obtained by averaging over the transmitted data to avoid the inverse matrix operation, and then (3) can be simplified as:

$$
\hat{H}_{M M S E}(l)=R_{H(l) H(l)}\left(R_{H(l) H(l)}+\frac{1}{S N R} I\right)^{-1} \hat{H}_{L S}(l),
$$

where

$$
S N R=\frac{E\left\{|X(l, k)|^{2}\right\}}{\sigma_{w}^{2}}
$$

Here, $I$ is an identity matrix, and $|\cdot|$ indicates the absolute value.

Second, a low-rank approximation is applied to a minimum mean-squared error (MMSE) estimator that uses the frequency correlation of the channel. To reduce the complexity, an optimal low-rank estimator derived from singular-value decomposition (SVD) is adopted [11]. The channel correlation matrix is first decomposed as

$$
R_{H(l) H(l)}=U \Lambda U^{H},
$$

where $\Lambda$ is a diagonal matrix with singular values $\lambda_{0} \geq \lambda_{1} \geq \ldots \geq$ $\lambda_{\mathrm{N}-1}, U$ is a unitary matrix.

The best rank-m approximation of the estimator in (6) then becomes:

$$
\hat{H}_{\text {OLR-MMSE }}(l)=U\left[\begin{array}{cc}
\Delta_{p} & 0 \\
0 & 0
\end{array}\right] U^{H} \hat{H}_{L S}(l),
$$

where $\Delta_{p}$ is a diagonal matrix that contains these values:

$$
\delta(k)=\frac{\lambda(k)}{\lambda(k)+\frac{1}{S N R}}, k=0,1, \ldots, p-1
$$

\section{Estimator Complexity Issue}

A complexity comparative approach of the described estimators shows:

1) LS algorithm leads to $N$ complex multiplications;

2) According to these terms: the inverse of $R_{H(l) H(l)}+\frac{1}{S N R} I \quad, \quad$ the product of $R_{H(l) H(l)}\left(R_{H(l) H(l)}+\frac{1}{S N R} I\right)^{-1}$ and $\hat{H}_{L S}(l)$, the MMSE algorithm contributes to $N^{3}+2 N^{2}+N$ complex multiplications for each OFDM block;

3) For the OLR-MMSE estimator, the rank-p approximation of the MMSE estimator in equation (7) can be re-expressed as a sum of rank-1 matrices as follows:

$$
\hat{H}_{\text {OLR-MMSE }}(l)=\left(\sum_{k=1}^{p} \delta u_{k} u_{k}^{H}\right) \hat{H}_{L S}(l),
$$

where $u_{k}$ denotes the $k_{\text {th }}$ column vector in the matrix $U$. The vectors $u_{k}$ for $k=1,2, \ldots, p$, can be tracked by means of the PASTD algorithm [12] with a significant reduced complexity of $2 \times N \times p$ for each OFDM block. The linear combination of $\mathrm{p}$ vectors of length $N$ in (9) requires $N \times \mathrm{p}$ multiplications. Hence, the OLR-MMSE estimator leads to $3 \times N \times p+N$ complex multiplications, which is significantly reduced than the MMSE estimator.

\section{Simulation Results}

The multiband OFDM system channel estimation was simulated to evaluate and compare performance of the considered channel estimation. In all simulations we have used CM1 as channel model. Others parameters are given in Table I. The OFDM symbols has been transmitted in radio frequency $(\mathrm{RF})$ using the TFC within BG1. We evaluate the system performance in terms of bit error rate (BER) over QPSK modulation and DCM modulation.

Three channel estimation method, LS, MMSE and 
OLR-MMSE, are simulated and compared. The results are shown in Fig. 3 and Fig. 4; the simulations are done for MB-OFDM UWB system with QPSK modulation and DCM modulation.

\section{TABLE I: MB-OFDM UWB SYSTEMS PARAMETERS}

\begin{tabular}{ll}
\hline \hline Parameter & Value \\
\hline FFT Size & 128 \\
Cyclic Prefix (CP) & 32 \\
Pilot Spacing & 5 \\
NSD: Number of data carriers & 100 \\
NSP: Number of pilot carriers & 12 \\
NSG: Number of guard carriers & 10 \\
NST: Number of total subcarriers & $122(=\mathrm{NSD}+\mathrm{NSP}+\mathrm{NSG})$ \\
used & $4.125 \mathrm{MHz}(=528 \mathrm{MHz} / 128)$ \\
$\Delta \mathrm{F}:$ Subcarrier frequency spacing & $242.42 \mathrm{~ns}(=1 / \Delta \mathrm{F})$ \\
TFFT: IFFT/FFT period & $60.61 \mathrm{~ns}(=32 / 528 \mathrm{MHz})$ \\
TCP: Cyclic prefix duration & $9.47 \mathrm{~ns}(=5 / 528 \mathrm{MHz})$ \\
TGI: Guard interval duration & $312.5 \mathrm{~ns}(=\mathrm{TCP}+\mathrm{TFFT}+\mathrm{TGI})$ \\
TSYM: Symbol interval & QPSK/DCM \\
Constellation & $200 \mathrm{Mbps} / 480 \mathrm{Mbps}$ \\
Data Rate & $528 \mathrm{MHz}$ \\
BandWidth & \\
\hline \hline
\end{tabular}

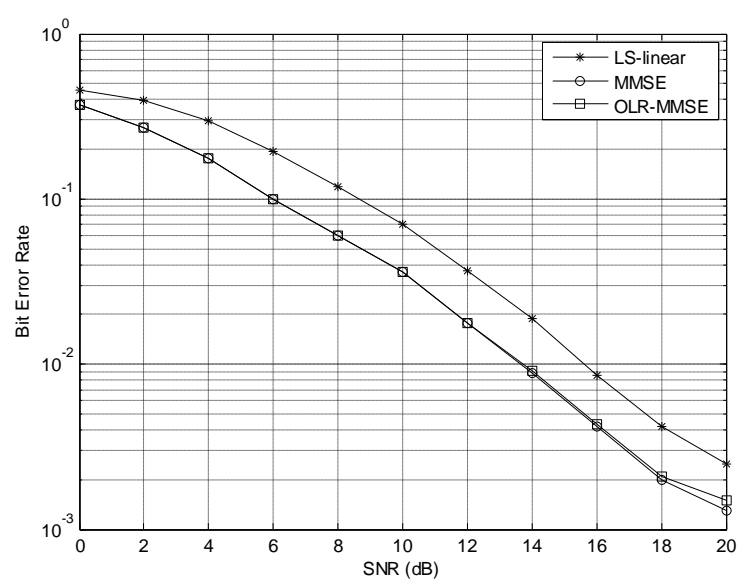

Fig. 3. BER for the channel estimators with QPSK modulation.

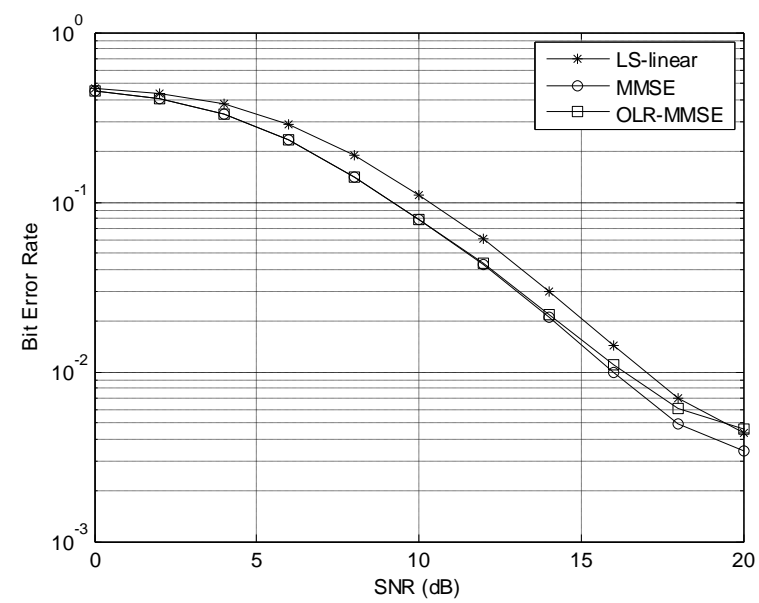

Fig. 4. BER for the channel estimators with DCM modulation.

The horizontal variable is signaling to noise ratio and the vertical variable is Bit Error Rate.
For both QPSK and DCM modulation (Fig. 3 and Fig. 4), OLR-MMSE estimator show comparable performance with MMSE estimator at low SNR and slightly under the MMSE estimator's performance at high SNR, on the other hand, has definitely better performance than the LS estimator.

Consequently, OLR-MMSE has been show to perform much better than LS and comparable performance with MMSE. Moreover, the complexity of MMSE is reduced by deriving an optimal low-rank estimator.

\section{CONCLUSION}

In this paper, we investigate the channel estimation techniques for MB-OFDM systems. The channel estimation based on comb-type pilot arrangement is studied through different algorithms for both estimating channel at pilot frequencies and interpolating the channel. The channel estimate at the pilot frequencies for comb-type based channel estimation can be based on LS or MMSE.

In this paper, the complexity of MMSE is reduced by deriving an optimal low-rank estimator OLR-MMSE. The simulation results show that the bit error rate (BER) performance significantly improved over Least Square (LS) Interpolation, and had comparable BER with MMSE channel estimation especially at low SNR with significant decrease in computational complexity.

We have compared the performances of all schemes by measuring bit error rate with DCM and QPSK, as modulation Schemes.

Finally is expected to improve these performances by combining the comb-type pilot based channel estimation with low-pass interpolation scheme.

\section{REFERENCES}

[1] J. Foerster, E. Green, S. Somayazulu, and D. Leeper, "Ultra-wideband technology for short- or medium-range wireless communications," Intel Technology JournalQ2, vol. 3, no. 6, 2001.

[2] WiMedia Alliance. [Online]. Available: http://www.wimedia.org/en/index.asp

[3] M. G. D. Benedetto, T. Kaiser, A. F. Molisch, I. Oppermann, C. Politano, and D. Porcino, UWB Communication Systems a Comprehensive Overview, Hindawi Publishing Corporation, May 2006

[4] S. M. R. Islam and K. S. Kwak, "On channel estimation in MB-OFDM UWB systems with time varying dispersive fading channel," International Journal of Digital Content Technology and its Applications, vol. 4, no. 2, April 2010

[5] S. Coleri, M. Ergen, A. Puri et al., "A study of channel estimation in OFDM systems," in Proc. IEEE 56th Vehicular Technology Conference, vol. 2, 2002, pp. 894-898.

[6] P. K. Pradhan, O. Fausty, S. K. Patra, and B. K. Chua, "Channel estimation algorithms for OFDM systems," International Journal of Signal and Imaging Systems Engineering, vol. 5, no. 4, pp. 267-273, November 2012.

[7] A. Chinchanikar and R. S. Kawitkar, "Overview: MB-OFDM UWB system," VSRD-IJEECE, vol. 2, no. 6, pp. 314-321, 2012

[8] R. F Yang and R. S.Sherratt, "Multiband OFDM modulation and demodulation for ultra wideband communications," Novel Applications of the UWB Technologies, pp. 1-30, 2007.

[9] W. Alliance, Multiband OFDM Physical Layer Specification, Technical report, MBOA, 2009.

[10] J. Foerster et al., "Channel Modeling Sub-Committee Report Final," tech. rep., IEEE P802.15-02/368r5-SG3a, July 2004.

[11] O. Edfors, J. J. Van D. Beek, S. K. Wilson, and P. O. Borjesson, "OFDM Channel Estimation by Singular Value Decomposition," IEEE Transactions On Communications, vol. 46, no. 7, pp. 931-939, July 1998.

[12] R. Landqvist and A. Mohammed, "The projection approximation subspace tracking algorithm applied to whitening and independent 
component analysis in wireless communications," Blekinge Institute of Technology Research Report, 2005

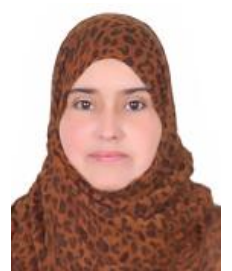

Moufida Hajjaj received her engineering degree in telecommunications in 2006 and the M.S degree in communications systems in 2007 from the National Engineering School of Tunis, Tunisia. Currently, she is a Ph.D student with the Innov'COM Laboratory research in telecommunication at Higher School of Communications of Tunis, Tunisia. She is also an assistant at the higher institute of management (ISG) of Gabes, Tunisia. Her research interests include channel estimation, OFDM, wireless systems, and ultra wideband systems.

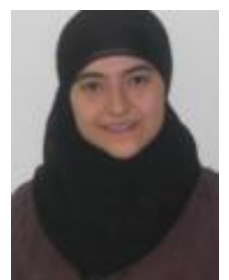

Fadoua Mhiri received her engineering degree in telecommunications in 2004, the M.S degree in communications systems in 2006 from the National Engineering School of Tunis, Tunisia and the Ph.D degree in telecommunications in 2013 from the National Engineer School of Tunis (ENIT), Tunisia. Since September 2013, she was an assistant professor in the Faculty of sciences of Sidibouzid. Her current research interests include wireless systems, high-speed networks and intelligence in networking.

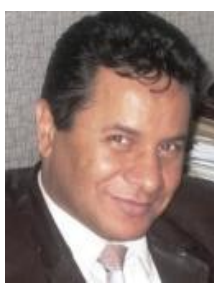

Ridha Bouallegue received his $\mathrm{Ph} . \mathrm{D}$ degree in electronic engineering from the National Engineering School of Tunis. In Mars 2003, he received the Hd.R degree in multiuser detection in wireless communications. From September 1990 he was a graduate professor in the higher school of communications of Tunis (SUP'COM), he has taught courses in communications and electronics. From 2005 to 2008, he was the director of the National engineering school of Sousse. In 2006, he was a member of the national committee of science technology. Since 2005, he was the Innov'COM laboratory research in telecommunication Director's at SUP'COM. From 2005 , he served as a member of the scientific committee of validation of thesis and Hd.R in the higher engineering school of Tunis. His current research interests include wireless and mobile communications, OFDM, space-time processing for wireless systems, multiuser detection, wireless multimedia communications, and CDMA systems. 